\title{
ATCA 13 AND 20 CM OBSERVATIONS OF A3556
}

\author{
S. BARDELLI ${ }^{1}$, R. MORGANTI ${ }^{2,3}$, T. VENTURI $^{2}$, \\ AND R.W. HUNSTEAD ${ }^{4}$ \\ 1 Osservatorio Astronomico di Trieste - Italy \\ ${ }^{2}$ Istituto di Radioastronomia, CNR-Bologna, Italy \\ 3 CSIRO-ATNF, Epping - Australia \\ ${ }^{4}$ University of Sydney - Australia
}

\section{A3556 and the Radio Observations}

We observed the cluster of galaxies A3556 ( $<v\rangle=14300 \mathrm{~km} / \mathrm{sec}$ ), belonging to the supercluster of galaxies known as the Shapley Concentration (Bardelli et al., 1994, MNRAS 267, 255, and references therein), with the Australia Telescope Compact Array. Our observations took place in the continuum band at $20 \mathrm{~cm}$ with the configurations $1.5 \mathrm{D}$ and $6 \mathrm{C}$, and at 20 $\mathrm{cm}$ and $13 \mathrm{~cm}$ in the configuration $6 \mathrm{~A}$, for a total of $12 \times 3$ hours, with a resolution ranging from $\sim 6^{\prime \prime}$ to $\sim 40^{\prime \prime}$. We observed a sky region of $\sim 2^{\circ} \times 1^{\circ}$ around the cluster center taking advance of the mosaicing technique developed ATNF.

These observations are part of a larger project, whose main aim is to study the interaction and evolution of clusters of galaxies located in dynamically unrelaxed environments, as is the case for A3556, deriving informations from their radio emission.

\section{Radio Emission from the Cluster Center}

The radio emission in A3556 is mainly concentrated around its optical center (see Fig. 1), characterised by a cD galaxy with an apparent magnitude $\mathrm{m}_{V}=14.4$ and by a few other luminous galaxies. Most of the bright members in the cluster have unresolved associated radio emission.

The most prominent feature is the extended radio source J1324-31. The appearance of this radio source is very unusual. Our maps at various resolu- 


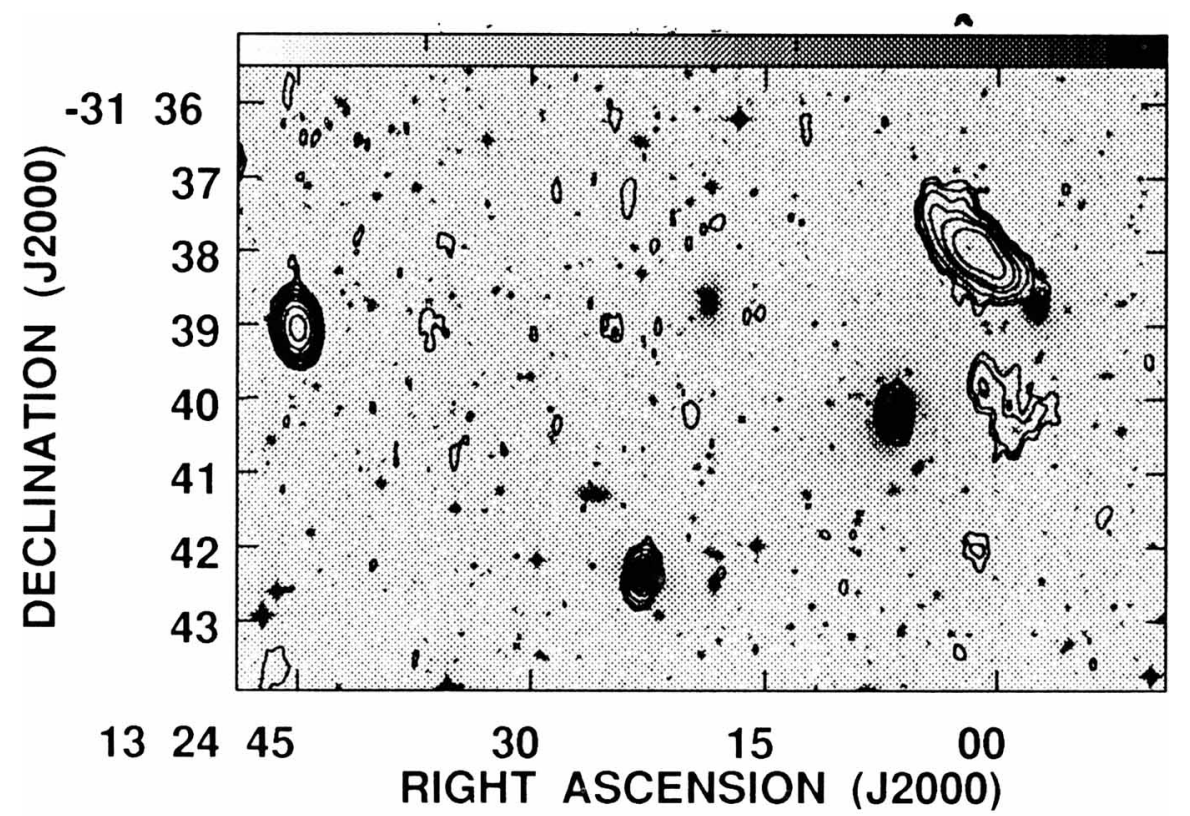

Figure 1. $20 \mathrm{~cm}$ image of the central region of A3556 superimposed on the DSS. The restoring beam is is $25.8^{\prime \prime} \times 15.6^{\prime \prime}$ mas, in p.a. $-1.21^{\circ}$. Peak flux is $29.3 \mathrm{mJy} / \mathrm{beam}$, levels $-0.3,0.3,0.5,1,2,3,5,10,20 \mathrm{mJy} /$ beam.

tions and frequencies show that J1324-31 has a very low surface brightness, and an amorphous morphology, without compact features.

The nature of this source is still unclear and we have been investigating it. J1324-31 could be either a cluster radio source or a background object. Two cluster galaxies are located very close to the radio emission, a 15.6 magnitude elliptical galaxy $(\langle v\rangle=15022 \mathrm{~km} / \mathrm{s})$, coincident with the far south end of the radio emission (see Fig. 1), and a 17.9 galaxy $(\langle v\rangle=$ $15142 \mathrm{~km} / \mathrm{s}$ ), located just outside the radio emission. A few other objects, without measured redshift, lie within the radio emission.

Assuming that the source is located at the cluster distance, its projected dimensions on the plane of the sky are $\sim 80 \times 25 \mathrm{kpc}\left(\mathrm{H}_{0}=100 \mathrm{~km}\right.$ $\left.\mathrm{s}^{-1} \mathrm{Mpc}^{-1}, \mathrm{q}_{0}=0\right)$ and its monochromatic power at $20 \mathrm{~cm}$ is $\log \mathrm{P}=23.05$. If we assume that equipartition holds within the source, then our $20 \mathrm{~cm}$ and $13 \mathrm{~cm}$ observations indicate that the equipartition magnetic field in the source is $\mathrm{H}_{e q} \sim 1 \mu \mathrm{G}$ and the minimum energy density is $\mathrm{U}_{\min } \sim 10^{-13}$ erg $\mathrm{cm}^{-3}$.

A detailed study of J1324-31 as well as of the global radio properties of A3556 is in progress. 